\title{
Strontium Promotes Cementoblasts Differentiation through Inhibiting Sclerostin Expression In Vitro
}

\author{
Xingfu Bao, ${ }^{1}$ Xianjun Liu, ${ }^{2}$ Yi Zhang, ${ }^{1}$ Yue Cui, ${ }^{1}$ Jindan Yao, ${ }^{1}$ and Min $\mathrm{Hu}^{1}$ \\ ${ }^{1}$ Department of Orthodontics, School of Stomatology, Jilin University, 1500 Qinghua Road, Changchun, Jilin 130021, China \\ ${ }^{2}$ Institute of Virology and AIDS Research, First Hospital, Jilin University, Changchun 130021, China \\ Correspondence should be addressed to Min Hu; humin@jlu.edu.cn
}

Received 2 January 2014; Revised 13 March 2014; Accepted 12 April 2014; Published 9 June 2014

Academic Editor: Kunikazu Tsuji

Copyright (c) 2014 Xingfu Bao et al. This is an open access article distributed under the Creative Commons Attribution License, which permits unrestricted use, distribution, and reproduction in any medium, provided the original work is properly cited.

\begin{abstract}
Cementogenesis, performed by cementoblasts, is important for the repair of root resorption caused by orthodontic treatment. Based on recent studies, strontium has been applied for osteoporosis treatment due to its positive effect on osteoblasts. Although promising, the effect of strontium on cementoblasts is still unclear. So the aim of this research was to clarify and investigate the effect of strontium on cementogenesis via employing cementoblasts as model. A series of experiments including MTT, alkaline phosphatase activity, gene analysis, alizarin red staining, and western blot were carried out to evaluate the proliferation and differentiation of cementoblasts. In addition, expression of sclerostin was checked to analyze the possible mechanism. Our results show that strontium inhibits the proliferation of cementoblasts with a dose dependent manner; however, it can promote the differentiation of cementoblasts via downregulating sclerostin expression. Taking together, strontium may facilitate cementogenesis and benefit the treatment of root resorption at a low dose.
\end{abstract}

\section{Introduction}

Cementum is a type of special mineralized tissue lying on the surface of teeth roots and has an analogous chemical element constituent to bone $[1,2]$. Due to its distinct position, cementum can connect with alveolar bone via fibers to maintain the position of teeth roots. Besides the function of roots anchoring, cementum is also believed to participate in the metabolic balance of dental tissues executed by cementoblasts [3-5]. This mentioned process is named as cementogenesis, which is often observed in periodontal tissue regeneration. One of such cases is the repair of root resorption origin from orthodontic treatment [6]. As a common adverse outcome in orthodontic treatment, root resorption usually occurred in more than $90 \%$ of orthodontic patients in clinic. Patients often endured root damage ranging from a slight loss of apical cementum to a total disappearance of root $[7,8]$. When orthodontic force is unloaded, cementoblasts lying on the surface of roots start to repair the resorption lacuna. Although the etiology of root resorption is still unclear, cementum has been regarded as an essential part to prevent the resorption $[9,10]$. What is more, cementogenesis has been well accepted as a target to alleviate root resorption currently. Thereby, various studies have been carried out to enhance the cementogenesis process [11-13].

As an effective antiosteoporosis drug, strontium ranelate can increase bone formation and inhibit bone resorption $[14,15]$. Recent studies have demonstrated that strontium as the bioactive component of this drug could stimulate the proliferation and differentiation towards osteogenic direction in mesenchymal cells and osteoblasts $[16,17]$. The mechanisms of strontium action are divided into calcium sensing receptor- (CaR-) dependent and CaR-independent signals. In the former one, strontium can act with $\mathrm{CaR}$ locating on the cell membrane and trigger different downstream pathways in osteoblast and osteoclast separately. Thus strontium can promote osteoblast replication, differentiation, and survival as well as reduce osteoclast differentiation, activity, and survival. In the latter one, some other signals such as Wnt signaling, OPG/RANKL signaling, and FGF/FGF receptor systems are involved [18]. Based on these dual activities on bone metabolism, strontium-contained drugs have been 
TABLE 1: Primers used for quantitative real-time PCR.

\begin{tabular}{lcc}
\hline Primers & Forward & Reverse \\
\hline Runx-2 & CTTCATTCGCCTCACAAAC & CTAGCAGTGACGGTCT \\
OCN & TGAACAGACTCCGGCG & GATACCGTAGATGCGTTTG \\
BSP & GAGACGGCGATAGTTCC & AGTGCCGCTAACTCAA \\
OPN & TTTACCAGCCTGCACCC & CTAGCAGTGACGGTCT \\
GAPDH & ACCACAGTCCATGCCATCAC & TCCACCACCCTGTTGCTGTA \\
\hline
\end{tabular}

successfully applied in clinic to reduce the risk of fracture in osteoporosis patients and improve the bone healing $[19,20]$.

Growing bodies of evidences have indicated the characteristics similarity between osteoblasts and cementoblasts; however, some major differences are still existent between cementum and bone. For example, there is no typically vascular system and lamellar organization in cementum. Otherwise, biological regeneration is of absence for cementum [21]. These discrepancies indicated the potential differences in metabolism between cementoblasts and osteoblasts under similar periodontal environment. Thereby it is meaningful and interesting to clarify the response of cementoblasts towards the strontium, which will substantiate the feasibility of strontium for root resorption therapy. Herein, influence of strontium on the proliferation and differentiation of cementoblasts were checked in detail. At the same time, expression of sclerostin, an endogenous antagonist for Wnt signaling, was examined to investigate the possible mechanism involved.

\section{Materials and Methods}

2.1. Cell Culture. The immortalized murine cementoblasts cell line (OCCM-30) was a gift from professor Ding Bai (Sichuan University, Department of Orthodontics, Chengdu, China). In accordance with previous reports, OCCM-30 cells between 20 and 25 passages with fine capacity of differentiation were used in this research. The OCCM-30 cells were cultured in DMEM supplemented with $10 \%$ fetal bovine serum (FBS). The culture media contained $100 \mathrm{UI} / \mathrm{mL}$ penicillin and $100 \mathrm{UI} / \mathrm{mL}$ streptomycin. The cell lines were cultured in a humidified atmosphere containing $5 \% \mathrm{CO}_{2}$ at $37^{\circ} \mathrm{C}$.

2.2. Proliferation Analysis. Cells were seeded in 96-well plates at a density of $4 \times 10^{3} /$ well in $\alpha$-MEM containing $1 \%$ FBS. After cell attachment, the media were changed to $\alpha$ MEM with strontium of different concentrations. After $24 \mathrm{~h}$ incubation, $10 \mu \mathrm{L}$ MTT $(5 \mathrm{~g} / \mathrm{L})$ was added to each well and cultured for another $4 \mathrm{~h}$. Then supernatant was removed and $150 \mu \mathrm{L}$ DMSO was added. It was shaken for $15 \mathrm{~min}$ for the crystal dissolution. The absorbance at $490 \mathrm{~nm}$ was measured with a micro-ELISA reader (Synergy 2, BioTek, Winooski, VT, USA). The proliferation rate was calculated compared to the control group.

2.3. Staining of Cell. $6 \times 10^{3}$ cells per well were cultured overnight in 6-well plates and incubated together with strontium at $12.5 \mu \mathrm{M}$ for $24 \mathrm{~h}$. Living cells were evaluated for morphology differences by employing calcein-AM staining. Generally, cells were washed twice with PBS (phosphate-buffered saline, $\mathrm{pH} 7.4)$ before calcein-AM $(10 \mathrm{ng} / \mathrm{mL})$ was added. After incubation for $20 \mathrm{~min}$ at $4^{\circ} \mathrm{C}$ in the dark, cells were observed using fluorescence microscopy (excitation (Ex), $365 \mathrm{~nm}$ and emission (Em), $480 \mathrm{~nm}$ ).

2.4. Gene Analysis by Real-Time PCR. Cementoblasts cultured in 6-well plates were incubated with strontium at $12.5 \mu \mathrm{M}$ for 3 days. Total RNA was extracted using RNAiso Plus (Takara Co., Japan) according to the manufactures' instructions. Then RNA was analyzed for quality and quantity by measuring the A260/A280 ratio with ultraviolet spectrophotometry. $2 \mu \mathrm{g}$ of total RNA was used in following reverse transcription reaction by employing PrimeScript RT reagent kit (Takara Co., Japan). Then each sample was analyzed by quantitative real-time PCR (qPCR) (Stratagene MX3000P, Japan) in the SYBR Premix Ex TapII (Takara Co., Japan), setting the cycles as follows: $10 \mathrm{~s} / 95^{\circ} \mathrm{C}$ PCR initial activation step, 40 cycles of denaturation for $20 \mathrm{~s} / 95^{\circ} \mathrm{C}$, and annealing step for $20 \mathrm{~s} / 60^{\circ} \mathrm{C}$. Formula $2^{-(\Delta \Delta \mathrm{CT})}$ was used to determine the change in mRNA levels, where $\Delta C T$ is the value from the threshold cycle (CT) of the treated sample subtracted from the CT value of untreated or zero timepoint control samples. Normalization to GAPDH mRNA was performed to decide the relative amount of mRNA in the sample. Primers used were listed in Table 1.

2.5. ALP Assay. Cells were seeded in 24-well plate at the density of $2 \times 10^{4}$ cells/well. After cell attachment, the culture medium was changed to $\alpha$-MEM, $10 \%$ FBS medium, and osteogenetic induction supplement containing $10 \mathrm{mmol} / \mathrm{L}$ disodium $\beta$-glycerophosphate and $0.15 \mathrm{mmol} / \mathrm{L}$ ascorbic acid. Strontium at $12.5 \mu \mathrm{M}$ was added to the culture medium of cells in treatment group. Then cells were incubated in the desired condition for 3 or 7 days followed by lysed using RIPA lysis buffer. Aliquots of supernatants were subjected to ALP activity and protein content measurement by an ALP activity kit and a microprotein assay kit (Jiancheng Biological Engineering Institute, Nanjing, China). ALP activities were normalized by total protein content.

2.6. Alizarin Red Staining. Cells were cultured in differentiation medium with or without $12.5 \mu \mathrm{M}$ strontium for 7 days. The formation of mineralized matrix nodules was determined by alizarin red staining. Briefly, the cells were fixed in 95\% ethanol for $30 \mathrm{~min}$ at room temperature. After PBS washing, alizarin red $(\mathrm{pH}=4.2)$ were added and incubated for $30 \mathrm{~min}$ at room temperature. Images of the staining results 


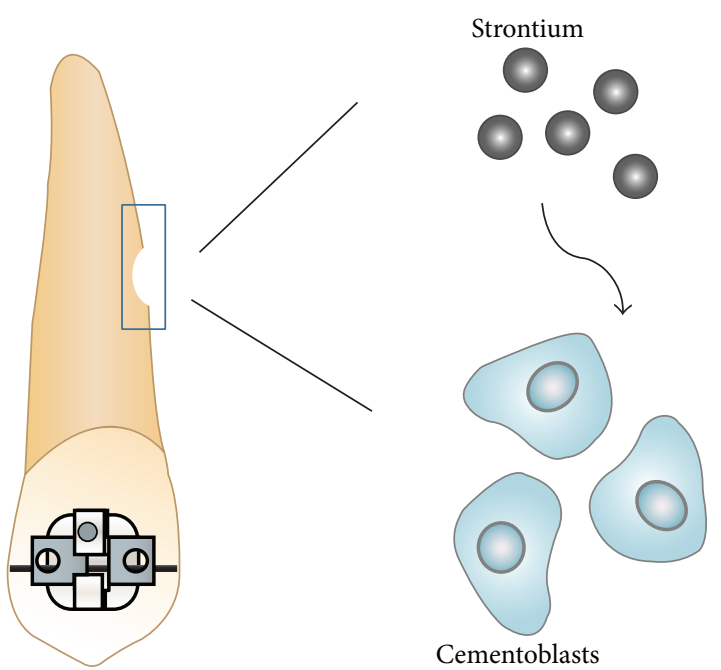

(a)
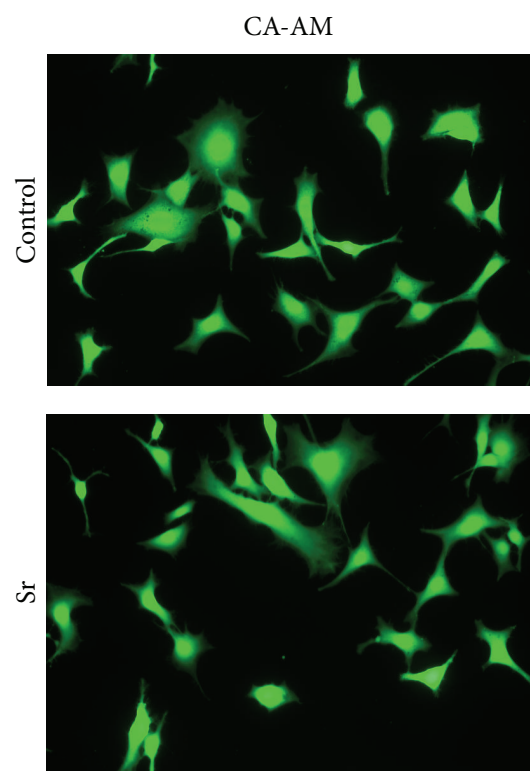

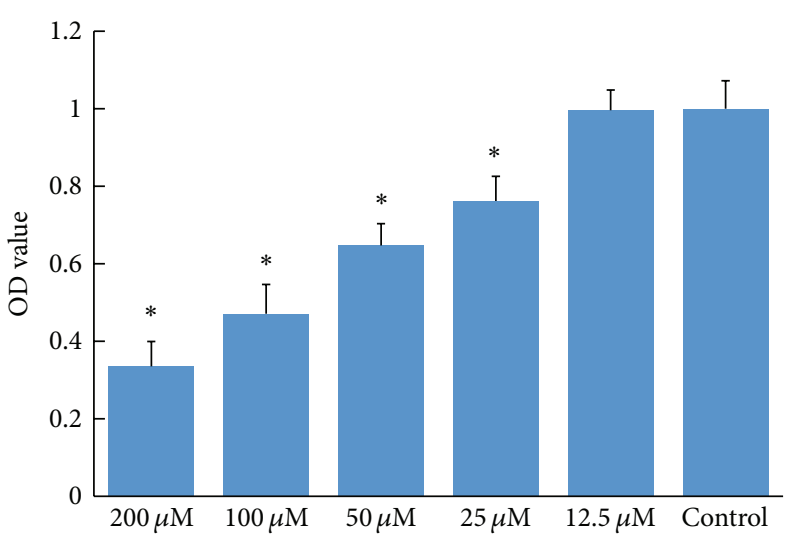

(b)
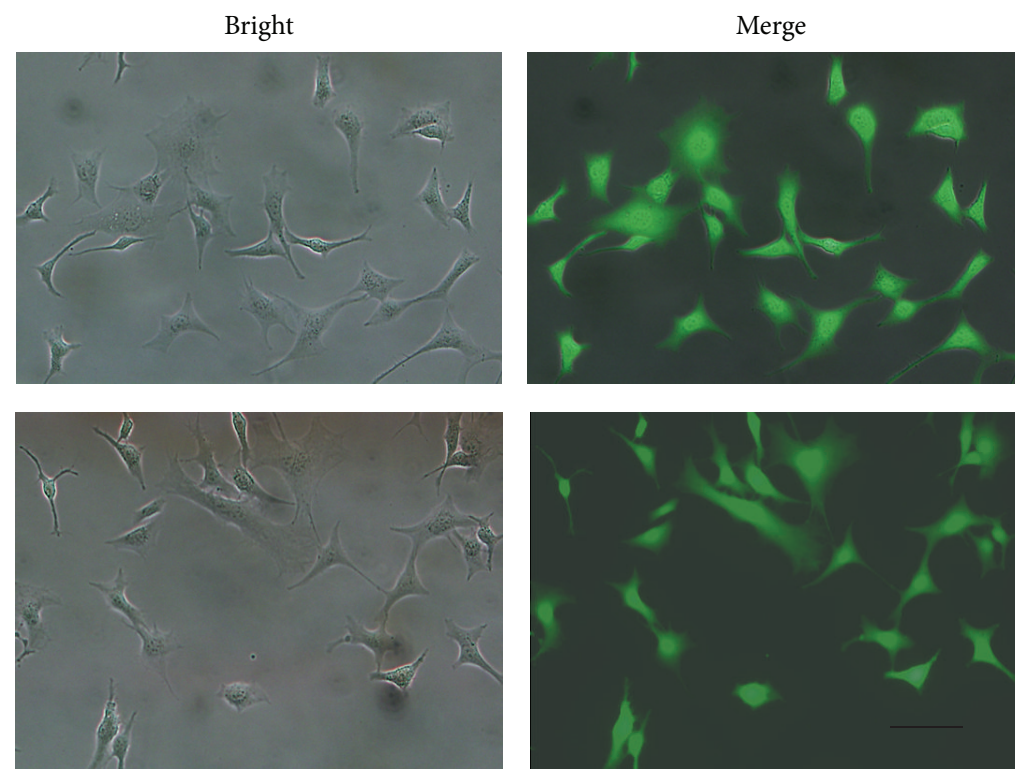

(c)

FIGURE 1: Schematic illustrating the modeling process of root resorption and the treatment of strontium (a). Cytotoxicity evaluated via MTT assays (b) and visible fluorescence microscopy images of OCCM-30 cells under different incubation conditions (c).

were recorded before quantitative analysis was performed. In detail, mineralization nodules were solved in $10 \%(\mathrm{w} / \mathrm{v})$ cetylpyridinium chloride for $10 \mathrm{~min}$ at room temperature followed by measurement of OD at $570 \mathrm{~nm}$. For mechanism analysis, recombinant human sclerostin (R\&D, USA) at concentration of $30 \mathrm{ng} / \mathrm{mL}$ was added to the osteogenic medium containing strontium.

2.7. Western Blot. After incubation with or without strontium at $12.5 \mu \mathrm{M}$ for 7 days, $40 \mu \mathrm{g}$ of total protein was separated by SDS-PAGE, using a gradient gel ((10-12\%), Bio-Rad Laboratories), transferred to nitrocellulose membrane, and analyzed by immunoblotting using the chemiluminescence (Santa Cruz, CA, USA). The primary antibodies used were sclerostin (Abcam, MA, USA, 1:500) or GAPDH (Santa Cruz, CA, USA, 1:1000), peroxidase-conjugated anti-mouse IgG (Santa Cruz, CA, USA, 1:1000). ImageJ software was applied to compare the intensity of protein bands to control through quantifying.

2.8. Immunofluorescence Microscopy. After incubation with or without strontium at $12.5 \mu \mathrm{M}$, cells on coverslips were fixed with $10 \%$ formalin in phosphate-buffered saline (PBS), $\mathrm{pH}$ 7.3 for $10 \mathrm{~min}$, and then permeabilized with $0.2 \%$ Triton $\mathrm{X}$ 100 in PBS for $5 \mathrm{~min}$ at room temperature. After blocking the cells with $1 \%$ skimmed milk for $1 \mathrm{~h}$ at room temperature, the cells were incubated with polyclonal mouse antisclerostin (Abcam, USA) antibodies at a 1:100 dilution overnight at 


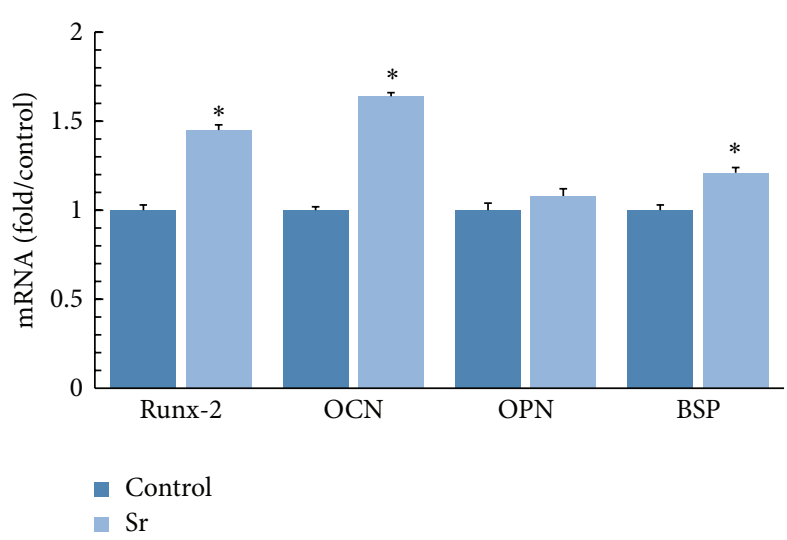

(a)

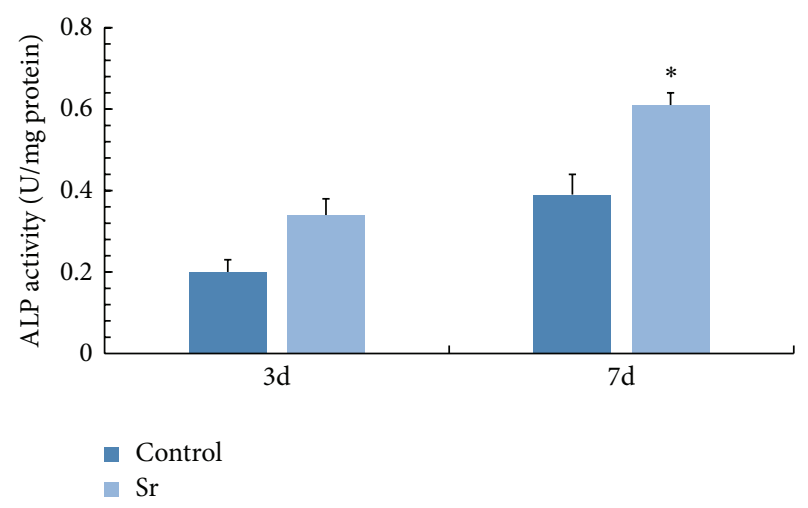

(b)

FIGURE 2: Expression of mineralization-related genes (a). ALP activity of cementoblasts after differentiation culture for 3 days and 7 days (b).

$4^{\circ} \mathrm{C}$. FITC-anti-mouse IgG (Santa Cruz, CA, USA) was used as a secondary antibody at a 1:200 dilution for $1 \mathrm{~h}$ at room temperature. Images were generated with a fluorescent microscope (BX 60, Olympus Co., Tokyo, Japan) operating a digital camera (DP 70, Olympus Co.).

2.9. Statistical Analysis. All experiments were performed thrice and the data were expressed as means $\pm \mathrm{SD}$. The difference between mean values was evaluated by using the ANOVA and considered to be statistically significant when $P<0.05$.

\section{Results}

3.1. Effect of Strontium Ions on the Proliferation of Cementoblasts. As shown in Figure 1(b), proliferation of cementoblasts is inhibited until the concentration of strontium ions decreased to $12.5 \mu \mathrm{M}$.

3.2. Analysis for the Morphology of Cementoblasts. Cementoblasts exhibited the normal triangular form when incubated with $12.5 \mu \mathrm{M}$ of strontium ions. As shown in Figure 1(c), there is no obvious change between the control group and treatment group.

3.3. Gene Analysis. After cementoblasts were incubated with strontium ions for 3 days, gene expressions of Runx-2, OCN, and BSP were upregulated significantly (Figure 2(a)), but it is nearly the same for OPN expression between two groups.

3.4. Analysis for ALP Expression. After cementoblasts were cultured in the presence of strontium, ALP activity increased and became significant from day 3 to 7 after differentiation (Figure 2(b)).

3.5. Regulation of Sclerostin Expression by Strontium Ions. Result of western blot analysis showed that strontium ions inhibit the expression of sclerostin in cementoblasts (Figures 3(a) and 3(b)). The immunofluorescence staining images presented in Figure 4 also indicated the same trend.

3.6. Alizarin Red Staining. Addition of strontium ions to the medium promoted the mineralization of cementoblasts, which was partially attenuated by recombinant sclerostin (Figures 3(d) and 3(e)). As presented in Figure 3(c), the quantitative analysis of alizarin red staining revealed the same trend.

\section{Discussions}

As a common side effect of orthodontic treatment, root resorption often brings orthodontists into a dilemma in clinical practice. To extricate from the trouble, numerous methods have been tried to cure the resorption. Previous studies have shown that cellular cementum constitutes the main pattern of repaired roots, which is formed by cementoblasts originated from periodontal cell precursors [9]. Therefore it is important to clarify the regulatory mechanisms of cementogenesis induced by cementoblasts. In addition, agents used to benefit bone formation will also affect the metabolism of cementum due to the similarity between bone and cementum. Being a potential promoting agent to bone disease, little is known related to the function of strontium in cementum regeneration (Figure 1(a)). In current study, we proved the effect of strontium on cementoblasts proliferation and differentiation by inhibiting sclerostin expression.

The effect of strontium on the proliferation of cementoblasts was checked firstly. Cementoblasts were treated with diverse concentrations of strontium ranging from $12.5 \mu \mathrm{M}$ to $200 \mu \mathrm{M}$ for 24 hours. As shown in Figure 1(b), no significant change was observed at the concentration of $12.5 \mu \mathrm{M}$. But when strontium concentration reached $200 \mu \mathrm{M}$, only 35\% of living cells were left compared to the control group, indicating the dose dependent inhibitory effect of strontium. This inhibition trend on proliferation is the same as previous studies performed on rat bone mesenchymal stem cells and osteoblasts $[16,22,23]$. But in some other reports, strontium is believed to promote cell proliferation at concentrations 


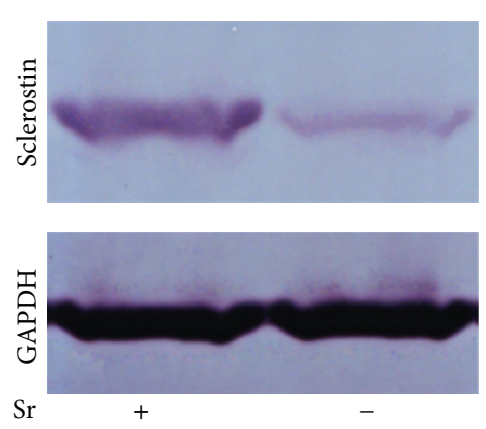

(a)
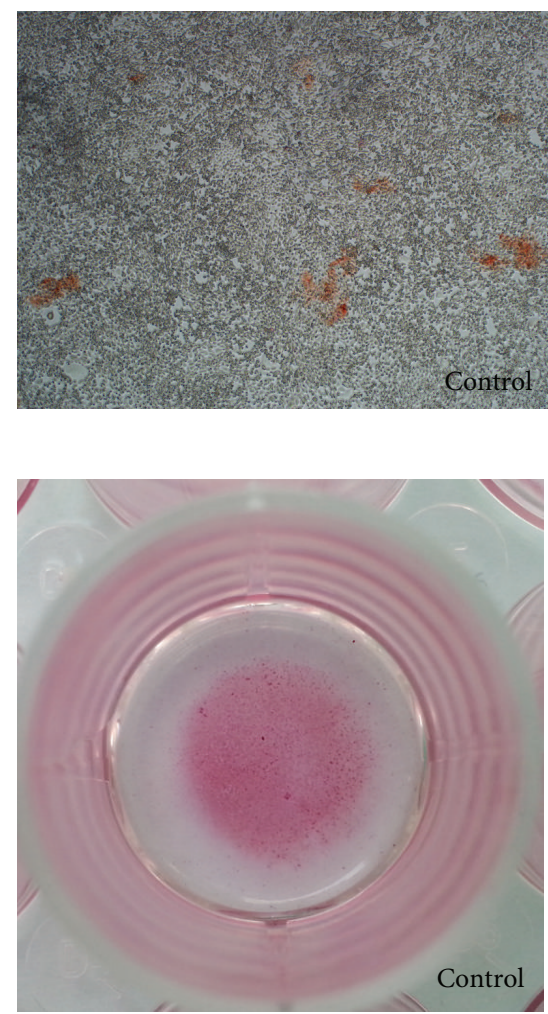

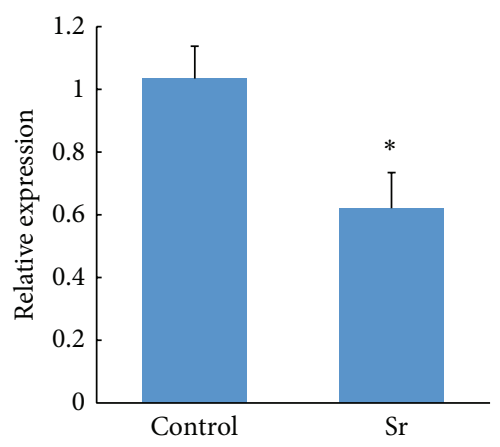

(b)

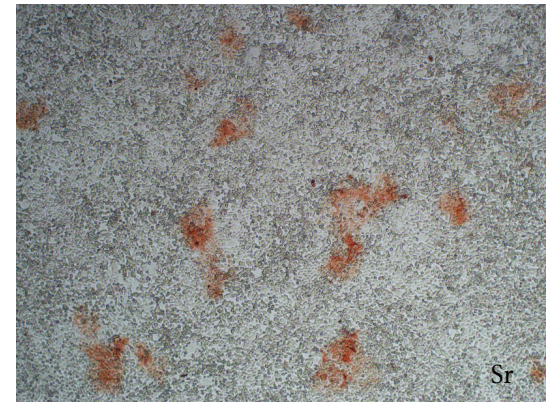

(d)
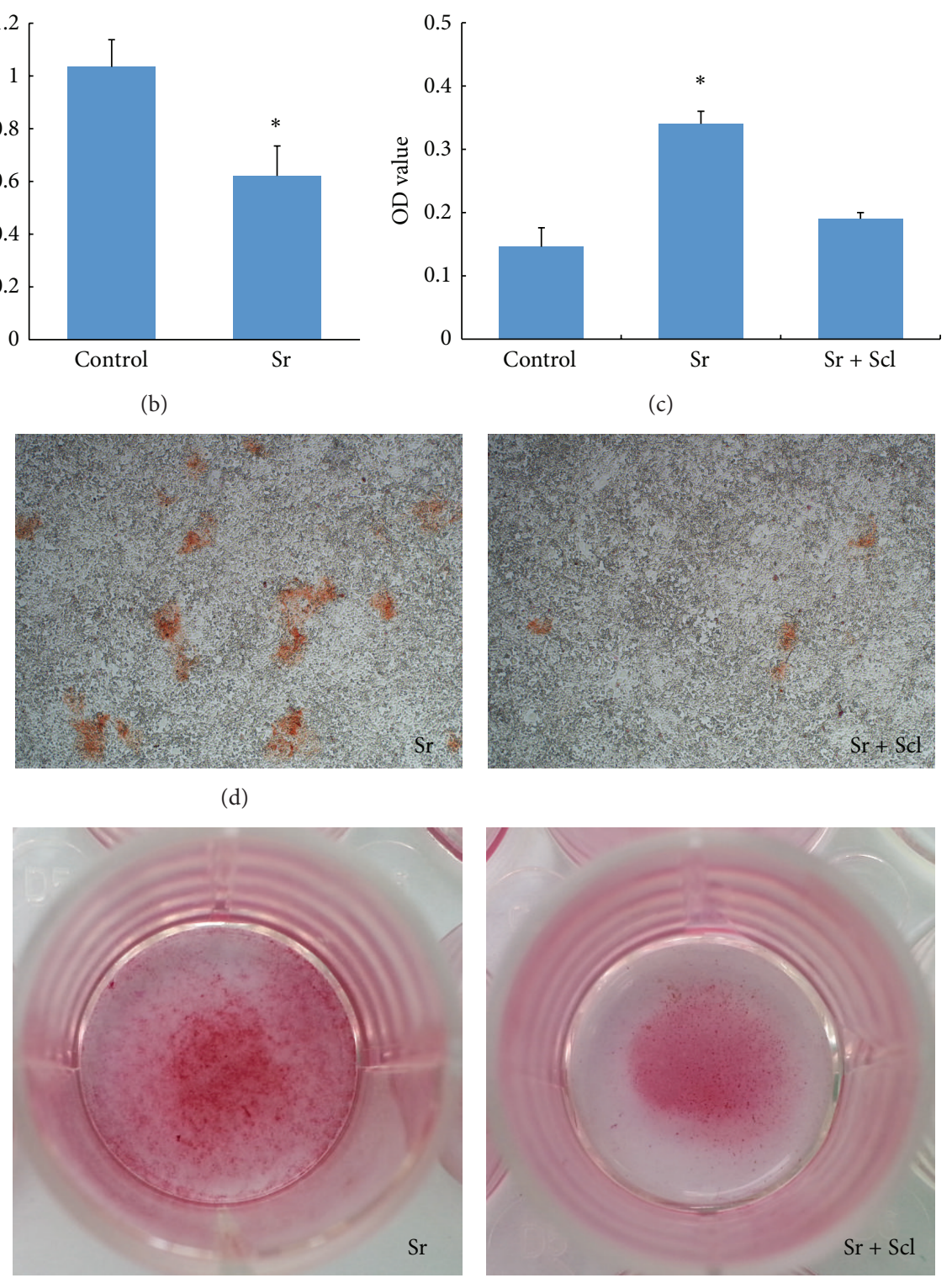

(c)

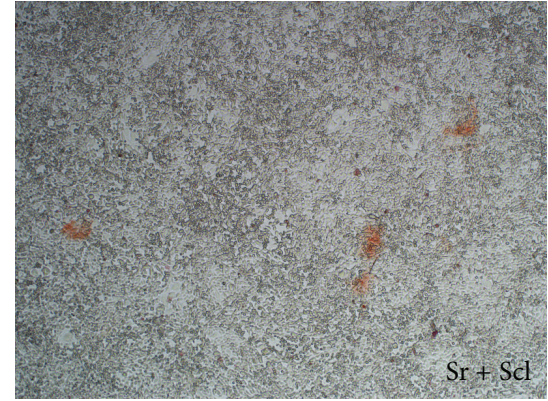

(e)

FIGURE 3: Expression of sclerostin after incubation with strontium ((a) and (b)). Alizarin red staining ((d) and (e)) and quantitative analysis (c) (Sr means strontium; Scl means recombinant human sclerostin).

higher than ours [24], which may be due to the different cell type and methods used in these researches. Since strontium ranelate is oral administration and has influence on the whole body, it is necessary to pay attention to the potential toxicity. From the perspective of toxicity, dosage of strontium used for osteoporosis treatment should be limited. Alternatively, local administration of strontium can be considered for the dental related usage.

Based on the results of proliferation analysis, we selected the lowest $12.5 \mu \mathrm{M}$ concentration to perform other experiments. Living-cell staining by calcein-AM could provide more visible information. As illustrated in Figure 1(c), cementoblasts demonstrated triangular form with protuberances and there were no obvious differences between 


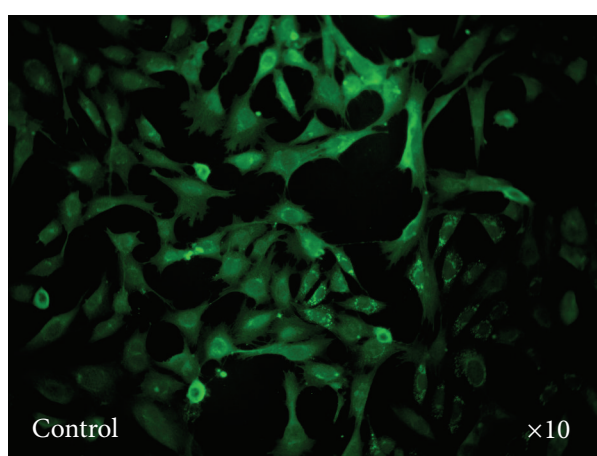

(a)

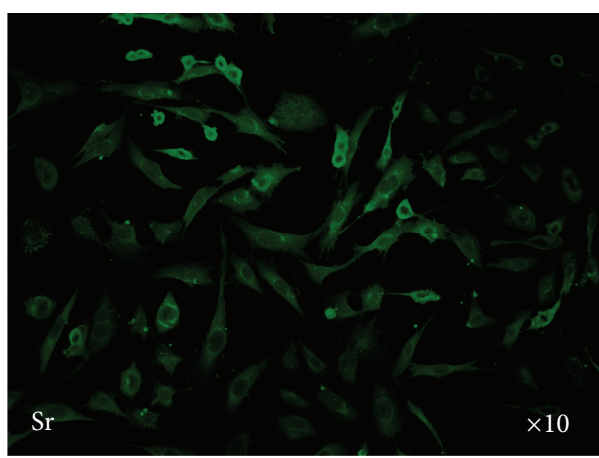

(c)

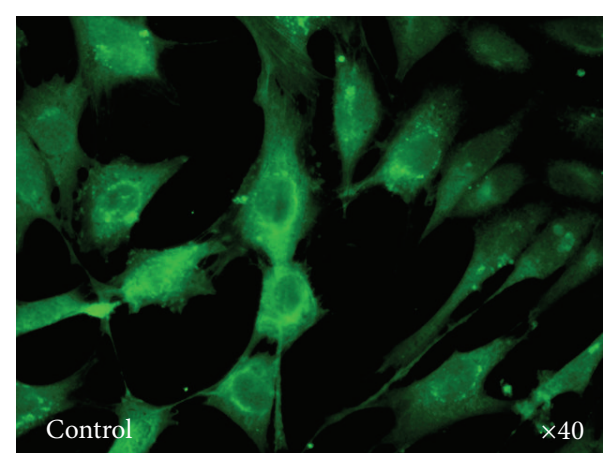

(b)

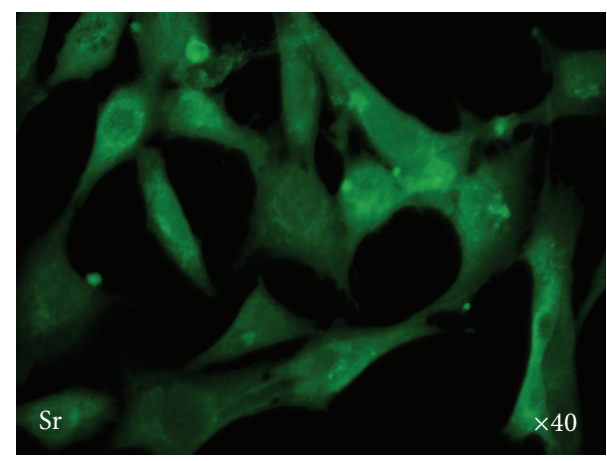

(d)

FIGURE 4: Immunofluorescence images of sclerostin in cementoblasts under different incubation conditions.

Noncollagenous proteins existing in cementum include BSP, ALP, dentin matrix protein 1 , osteopontin, and several growth factors. In common, Runx2 is regarded as a positive regulator of ALP, BSP, and OCN in the cementoblasts differentiation duration. OCN is a late marker for cementoblasts differentiation and participates in the mineral deposition. BSP, which is mainly lying on the root surface in cementogenesis, has been proved to trigger the mineralization and enhance the adhesion and differentiation of cementoblasts [26]. Therefore, gene analysis based on our study demonstrated that strontium might promote the differentiation of cementoblasts via upregulation of mineralization-related genes including Runx2, OCN, and BSP. As another important marker to describe the differentiation of cementoblasts, ALP expression was investigated after 3 days and 7 days of incubation. Figure 2(b) indicated that the ALP activity increased in the presence of strontium in a time-dependent manner. Alizarin red-S staining and OD value analysis were applied to evaluate the level of mineralization. As shown in Figures 3(c), 3(d), and 3(e), upon 7 days of incubation with strontium, redstained mineralized nodules could be clearly observed from the control group and treated group. Highly in accordance with other differentiation markers, more red nodules could be seen in the presence of strontium.

To further clarify the possible mechanism involved in the positive effect on the differentiation of cementoblasts, we analyzed the expression of sclerostin after incubation of 7 days. As shown in Figures 3(a) and 3(b), sclerostin protein expression in cementoblasts occurred in the osteogenic differentiation culture medium. Compared with that of the control group, supplement of strontium into the differentiation medium could significantly reduce the expression of sclerostin by $30 \%$. Otherwise, immunofluorescence analysis also indicated the above changes. In detail, stronger fluorescence could be observed in control group than in treated group (Figure 4). Encoded by SOST gene, sclerostin has been considered as an antagonist of Wnt signaling [27]. Because of the dual effect of sclerostin on bone turnover, the expression of sclerostin in dental tissue has attracted much more attention in recent years. For example, increasing the levels of sclerostin protein could be verified in the periodontal ligament cells after mineralization treatment [28]. In the present study, recombinant human sclerostin was further employed to investigate the speculation via the alizarin red staining. As described in Figures 3(c) and 3(d), enhanced mineralization induced by strontium could be shielded by the supplement of recombinant human sclerostin, indicating the inhibitory effect of sclerostin on the differentiation of cementoblasts [29].

\section{Conclusions}

In conclusion, we investigated the in vitro effect of strontium on the differentiation of cementoblasts via various experiments ranging from cell biology to molecular biology for the first time. Based on our results and other previous studies, one possible mechanism for above action was that strontium could inhibit the expression of sclerostin to promote the 
differentiation of cementoblasts. Thereby, strontium could be considered as a potential target to facilitate cementum repair in root resorption patients. However, due to the lower tolerance of cementoblasts towards strontium compared with osteoblasts and the complex environment in vivo, more studies were still highly needed before further application in clinic.

\section{Conflict of Interests}

The authors declare that there is no conflict of interests regarding the publication of this paper.

\section{Acknowledgments}

The authors thank Professor Somerman and Ding Bai for providing cell line to them. This work was supported by National Natural Science Foundation of China (NSFC) (81170999); Special Industrial Research supported by Development and Reform Commission of Jilin Province (2013C025-2); and Specialized Research Fund for Doctoral Program of Higher Education, China, SRFDP (20110061110072).

\section{References}

[1] D. D. Bosshardt and K. A. Selvig, "Dental cementum: the dynamic tissue covering of the root," Periodontology 2000, vol. 14, no. 1, pp. 41-75, 1997.

[2] A. Nanci and D. D. Bosshardt, "Structure of periodontal tissues in health and disease," Periodontology 2000, vol. 40, no. 1, pp. 11-28, 2006.

[3] D. D. Bosshardt, "Are cementoblasts a subpopulation of osteoblasts or a unique phenotype?" Journal of Dental Research, vol. 84, no. 5, pp. 390-406, 2005.

[4] Z. Artzi, N. Wasersprung, M. Weinreb, M. Steigmann, H. S. Prasad, and I. Tsesis, "Effect of suided tissue regeneration on newly formed Bone and cementum in periapical tissue healing after endodontic surgery: an in vivo study in the cat," Journal of Endodontics, vol. 38, no. 2, pp. 163-169, 2012.

[5] D. W. K. Kao and J. P. Fiorellini, "Regenerative periodontal therapy," Frontiers of Oral Biology, vol. 15, pp. 149-159, 2012.

[6] J.-H. Lee, J. D. Lin, J. I. Fong, M. I. Ryder, and S. P. Ho, “The adaptive nature of the bone-periodontal ligament-cementum complex in a ligature-induced periodontitis rat model," BioMed Research International, vol. 2013, Article ID 876316, 17 pages, 2013.

[7] N. Brezniak and A. Wasserstein, "Orthodontically induced inflammatory root resorption. Part II: the clinical aspects," Angle Orthodontist, vol. 72, no. 2, pp. 180-184, 2002.

[8] N. Brezniak and A. Wasserstein, "Orthodontically induced inflammatory root resorption. Part I: the basic science aspects," Angle Orthodontist, vol. 72, no. 2, pp. 175-179, 2002.

[9] A. Jäger, D. Kunert, T. Friesen, D. Zhang, S. Lossdörfer, and W. Götz, "Cellular and extracellular factors in early root resorption repair in the rat," European Journal of Orthodontics, vol. 30, no. 4, pp. 336-345, 2008.

[10] P. Owman-Moll, J. Kurol, and D. Lundgren, "Repair of orthodontically induced root resorption in adolescents," The Angle orthodontist, vol. 65, no. 6, pp. 403-410, 1995.
[11] E. B. Rego, T. Inubushi, A. Kawazoe et al., "Effect of PGE2 induced by compressive and tensile stresses on cementoblast differentiation in vitro," Archives of Oral Biology, vol. 56, no. 11, pp. 1238-1246, 2011.

[12] S. S. Hakki, B. L. Foster, K. J. Nagatomo et al., "Bone morphogenetic protein-7 enhances cementoblast function in vitro," Journal of Periodontology, vol. 81, no. 11, pp. 1663-1674, 2010.

[13] X. Bao, M. Hu, Y. Zhang et al., "Effect of fangchinoline on root resorption during rat orthodontic tooth movement," Korean Journal of Orthodontics, vol. 42, no. 3, pp. 138-143, 2012.

[14] S. Das and J. C. Crockett, "Osteoporosis-a current view of pharmacological prevention and treatment," Drug Design, Development and Therapy, vol. 7, pp. 435-448, 2013.

[15] Y. Wu, S. M. Adeeb, M. John Duke, D. Munoz-Paniagua, and M. R. Doschak, "Compositional and material properties of rat bone after bisphosphonate and/or strontium ranelate drug treatment," Journal of Pharmacy and Pharmaceutical Sciences, vol. 16, no. 1, pp. 52-64, 2013.

[16] Y. Li, J. Li, S. Zhu et al., "Effects of strontium on proliferation and differentiation of rat bone marrow mesenchymal stem cells," Biochemical and Biophysical Research Communications, vol. 418, no. 4, pp. 725-730, 2012.

[17] W. Querido and M. Farina, "Strontium ranelate increases the formation of bone-like mineralized nodules in osteoblast cell cultures and leads to Sr incorporation into the intact nodules," Cell and Tissue Research, vol. 354, no. 2, pp. 573-580, 2013.

[18] Z. Saidak and P. J. Marie, "Strontium signaling: molecular mechanisms and therapeutic implications in osteoporosis," Pharmacology and Therapeutics, vol. 136, no. 2, pp. 216-226, 2012.

[19] C. Wu, Y. Zhou, C. Lin, J. Chang, and Y. Xiao, "Strontiumcontaining mesoporous bioactive glass scaffolds with improved osteogenic/cementogenic differentiation of periodontal ligament cells for periodontal tissue engineering," Acta Biomaterialia, vol. 8, no. 10, pp. 3805-3815, 2012.

[20] M. Baier, P. Staudt, R. Klein et al., "Strontium enhances osseointegration of calcium phosphate cement: a histomorphometric pilot study in ovariectomized rats," Journal of Orthopaedic Surgery and Research, vol. 8, no. 1, article 16, 2013.

[21] X.-F. Huang and Y. Chai, "Molecular regulatory mechanism of tooth root development," International Journal of Oral Science, vol. 4, no. 4, pp. 177-181, 2013.

[22] I. Schrooten, G. J. S. Behets, W. E. Cabrera et al., "Dose-dependent effects of strontium on bone of chronic renal failure rats," Kidney International, vol. 63, no. 3, pp. 927-935, 2003.

[23] S. C. Verberckmoes, M. E. De Broe, and P. C. D'Haese, "Dosedependent effects of strontium on osteoblast function and mineralization," Kidney International, vol. 64, no. 2, pp. 534543, 2003.

[24] P. Römer, B. Desaga, P. Proff, A. Faltermeier, and C. Reicheneder, "Strontium promotes cell proliferation and suppresses IL-6 expression in human PDL cells," Annals of Anatomy, vol. 194, no. 2, pp. 208-211, 2012.

[25] D. D. Bosshardt, S. Zalzal, M. D. McKee et al., "Developmental appearance and distribution of bone sialoprotein and osteopontin in human and rat cementum," Anatomical Record, vol. 250, no. 1, pp. 13-33, 1998.

[26] S. S. Hakki, S. B. Bozkurt, E. E. Hakki, and S. Belli, "Effects of mineral trioxide aggregate on cell survival, gene expression associated with mineralized tissues, and biomineralization of cementoblasts," Journal of Endodontics, vol. 35, no. 4, pp. 513519, 2009. 
[27] M. J. Moester, S. E. Papapoulos, C. W. Löwik, and R. L. van Bezooijen, "Sclerostin: current knowledge and future perspectives," Calcified Tissue International, vol. 87, no. 2, pp. 99-107, 2010.

[28] A. Jäger, W. Götz, S. Lossdörfer, and B. Rath-Deschner, "Localization of SOST/sclerostin in cementocytes in vivo and in mineralizing periodontal ligament cells in vitro," Journal of Periodontal Research, vol. 45, no. 2, pp. 246-254, 2010.

[29] X. Bao, Y. Liu, G. Han et al., "The effect on proliferation and differentiation of cementoblast by using sclerostin as inhibitor," International Journal of Molecular Sciences, vol. 14, no. 10, pp. 21140-21152, 2013. 

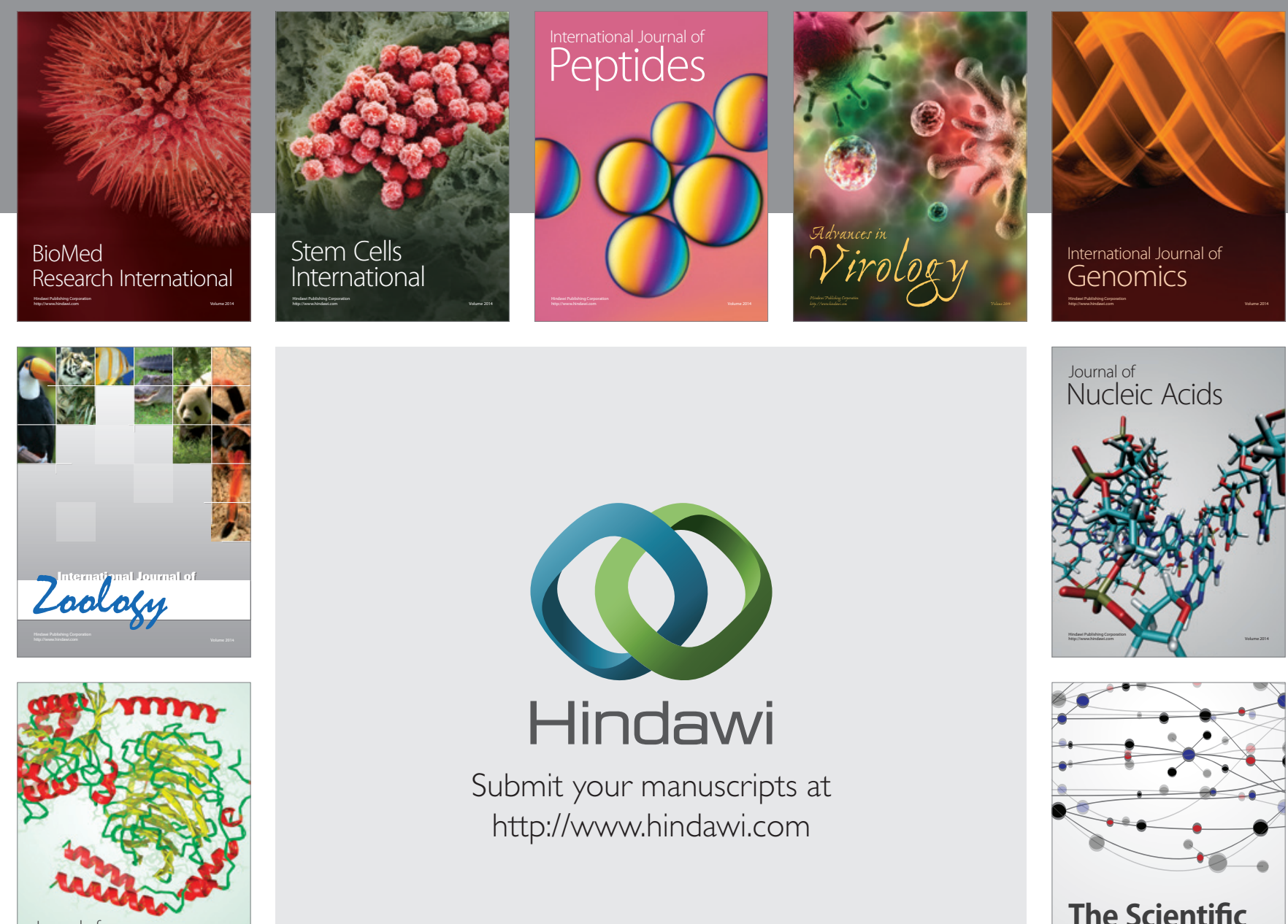

Submit your manuscripts at

http://www.hindawi.com

Journal of
Signal Transduction
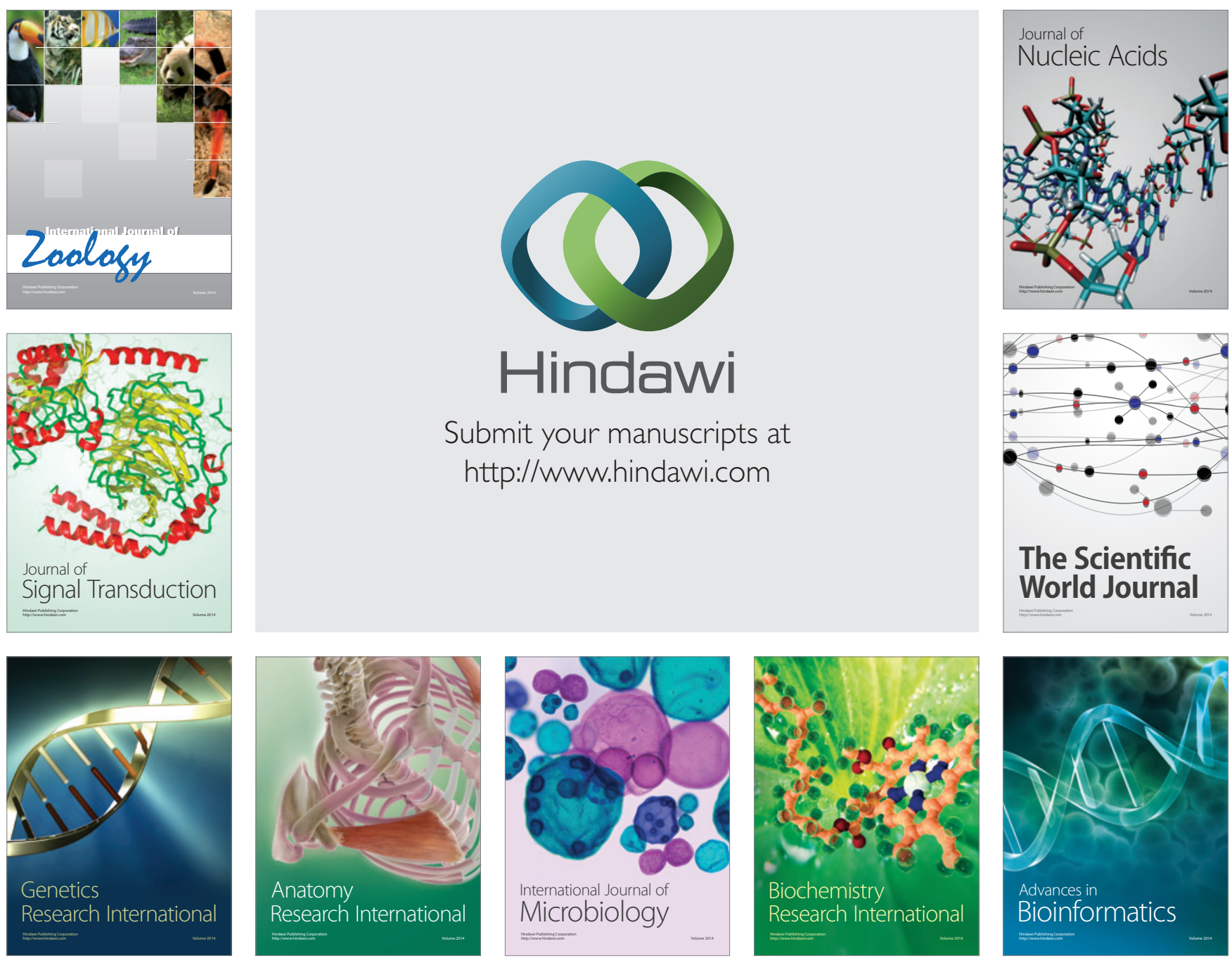

The Scientific World Journal
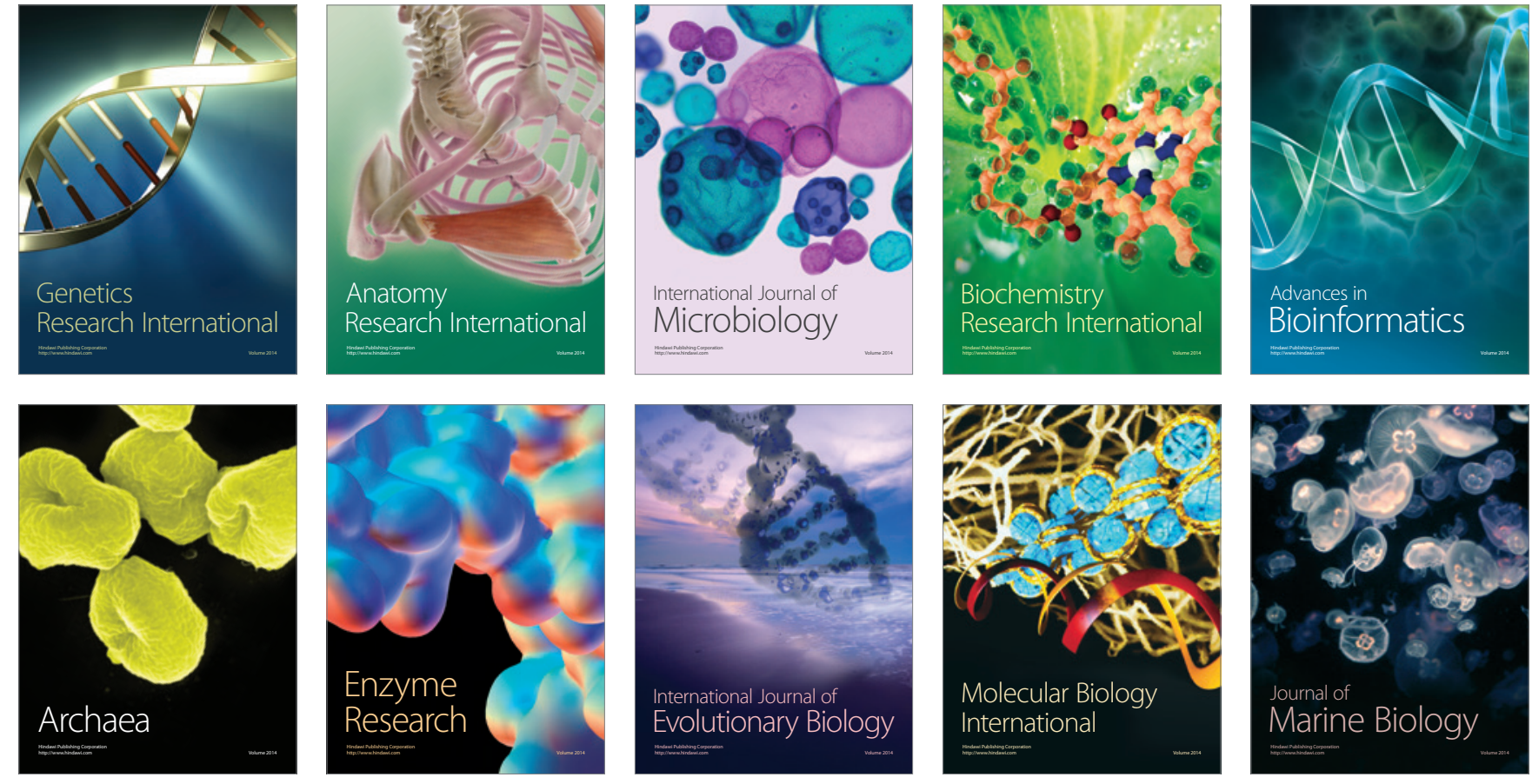